\title{
Motor simulation in tool-use effect on distance estimation: A replication of Witt and Proffitt (2008)
}

\author{
Lisa Molto $^{1} \cdot$ Nicolas Morgado $^{2} \cdot$ Eric Guinet ${ }^{1} \cdot$ Laurina Fazioli $^{1} \cdot$ Loïc P. Heurley $^{2} \cdot$ Richard Palluel-Germain $^{1}$
}

Published online: 21 January 2020

(C) The Psychonomic Society, Inc. 2020

\begin{abstract}
Witt and Proffit (Human Perception and Performance, 34 (6), 1479-1492, 2008) hypothesized that when people intend to reach a target, they run a motor simulation allowing them to anticipate potential reaching constraints and outcomes, which in turn affects spatial perception. They reported that participants estimated targets to be closer to them when they intended to use a reachextending tool, but only when they did not perform a concurrent motor task. The authors concluded that the concurrent motor task prevented the simulation of tool-use and its effect on perception. Reported here is a replication that extends their work through an additional control group and a larger sample size. Our results failed to support either the role of motor simulation in the tool-use effect on distance estimation or the tool-use effect itself. Moreover, a reanalysis of Witt and Proffitt's data suggested that they should have been more nuanced in their own conclusions. Further replications are needed in order to elucidate the existence, nature, boundary conditions, and underlying mechanisms of the action constraint effects on space perception.
\end{abstract}

Keywords Distance perception $\cdot$ Tool-use $\cdot$ Motor simulation $\cdot$ Registered replication report

\section{Introduction}

Studies have shown that people estimate targets to be closer to them when they are holding a tool that extends their reaching ability (Costello et al., 2015; Osiurak, Morgado, \& PalluelGermain, 2012; Witt, 2011; Witt \& Proffitt, 2008; Witt, Proffitt, \& Epstein, 2005). This effect may reflect that space perception depends on constraints associated with people's intended actions (Morgado \& Palluel-Germain, 2016; Witt, 2017). According to this view, the visual perception of objects requires a motor-based perceptual system combining visual and motor-related variables (Coello \& Delevoye-Turrell, 2007; Witt \& Proffitt, 2008). As such, people should perceive that an object is closer when they can act on it more easily. Witt and Proffitt (2008) argued that such an effect relies on motor simulation, which refers to the activation of common

Richard Palluel-Germain

richard.palluel@univ-grenoble-alpes.fr

1 University of Grenoble Alpes, University of Savoie Mont Blanc, CNRS, LPNC, Grenoble, France

2 University of Paris Nanterre, LICAE, Nanterre, France neural pathways when performing, imagining, or observing a given action (Jeannerod, 2001). Thus, when people intend to perform an action, they run a motor simulation of this action, allowing them to anticipate its potential constraints and outcomes, which in turn affects spatial perception.

Witt and Proffitt (2008) tested this idea with a motor interference procedure. In two studies, participants had to estimate the distance to a target located beyond arm reach, prior to performing a reaching movement toward this target. In one study, participants had to reach targets with a 39-cm-long baton, held in their right hand, making the targets reachable (Study 3a). In another study, participants had to try to reach the same targets with their right hand without the baton (Study 3b). In both studies, participants had to estimate the distance to the target with a visualmatching task. Using a keyboard with the left hand, they had to adjust the distance between two comparison points, until it visually matched the distance to the target. In each study, during this visual-matching task, one group of participants squeezed a rubber ball with their right hand whereas another group did not. This squeezing task was intended to recruit the motor system and should have therefore interfered with the motor simulation of reaching during distance estimation. The results indicated that the 
no-squeeze group estimated that the targets were closer than the squeeze group only when the participants could use the baton to reach the targets (Study 3a). According to the authors, people consider the advantages of reachextending tools by simulating their use, unless they are performing a concurrent motor task that interferes with motor simulation. They also concluded that this interference was effector-specific because the concurrent motor task with the left hand (using the keyboard) during the visual-matching task did not interfere with the anticipation of tool-use with the right hand.

These conclusions play a central role in the space perception literature, and Witt and Proffitt (2008)'s article is often cited to support the proposal that the motor system is involved in the distance perception (196 citations in Google Scholar; Median $_{\text {citations per papers in this literature }} \approx 35$ ). For this reason, and because it has not yet been conducted, we aimed to replicate these studies in order to improve the estimation of the moderator effect of the presence of motor interference. Moreover, Witt and Proffitt's conclusion needs to be qualified in the light of some methodological choices they made.

First, Witt and Proffitt (2008) compared one group performing a dual task (ball squeezing and distance estimation) to another group performing only a simple task (distance estimation). Thus, any differences between these groups could derive from the fact that the participants performed a concurrent task, regardless of the motor nature of this task. ${ }^{1}$ To overcome this eventuality, we added a control group in which participants performed a motorconcurrent task during distance estimation with an effector that was not involved in the subsequent reaching to the target (i.e., the left foot). This condition allows a proper test of the motor simulation hypothesis while keeping constant the number and the nature of the tasks that the participants performed at the same time.

Second, Witt and Proffitt (2008) did not randomly assign their participants either to the tool groups and the notool groups or to the squeeze and no-squeeze groups. Indeed, all the participants from the Study 3 a reached with a tool and all the participants from the Study $3 \mathrm{~b}$ reached without it. Moreover, in both studies, the authors alternately assigned their participants to the squeeze and no-squeeze groups. ${ }^{2}$ Compared to such non-random

\footnotetext{
${ }^{1}$ Witt and Proffitt (2008) tried to rule out this interpretation by arguing that the attentional demand of applying a constant squeeze on a rubber ball is negligible and because they did not observe the interference effect when participants did not intend to reach with the baton.

${ }^{2}$ Even if the authors did not explicitly mention they used alternated assignment for their Studies 3a and 3b, we considered that they did that based on what they did in their other studies (Witt \& Proffitt, 2008, p. 1485; e.g., Witt, 2011, p. 1150; Witt, Proffitt, \& Epstein, 2004, p. 580) and details they provided in a personal communication (J. K. Witt, personal communication, 28 May 2018).
}

assignments, ${ }^{3}$ random assignment makes conclusions less prone to threat of the internal validity and allows computation of unbiased estimates of effect size (e.g., Shadish, Cook, \& Campbell, 2002, pp. 247-252). Thus, in the present study, we randomly assigned our participants to the experimental groups.

Third, Witt and Proffitt (2008) concluded that motor interference influenced distance estimation only when participants intended to reach the target with a tool. This conclusion implies that motor interference moderates the effect of tool use on distance estimation. However, the authors based their conclusion on a statistically significant effect of motor interference in Study 3a and a non-significant effect in Study 3b, which is not an appropriate test for interaction effects (Gelman \& Stern, 2006).

Finally, the sample size of the original studies seems small to test the effects of interest (e.g., tool-use effect, Motor Interference $\times$ Tool-Use interaction). We conducted a smallscale meta-analysis combining nine estimates of tool-use effects on distance estimation (Bloesch, Davoli, Roth, Brockmole, \& Abrams, 2012; Costello et al., 2015; Osiurak et al., 2012; Witt, 2011; Witt \& Proffitt, 2008; Witt et al., 2005), resulting in an average estimate of Cohen's $d_{\text {unbiased }}=$ $0.34,95 \%$ confidence interval (CI) for Cohen's $\delta[0.12,0.55]$. We used the tool-use effect size as the minimum effect size of interest as data available in the paper (and from the authors) did not allow computation of a standardized effect size for the Presence of the Motor Interference $\times$ Tool Availability interaction. According to a statistical power analysis (G*Power 3, Faul, Erdfelder, Lang, \& Buchner, 2007), the research design used by Witt and Proffitt's (2008) Studies 3a and 3b only had a power of .15 to detect a tool-use effect on distance estimation with a Cohen's $\delta$ of 0.34 (for a two-tailed between-subject ttest, $\left.\alpha=.05, n_{\text {tool }}=n_{\text {no-tool }}=16\right) .{ }^{4}$ This means that the original studies were underpowered. To overcome this limitation, we used a more appropriate sample size in our replication.

The present study is a close replication of Witt and Proffitt's (2008) Studies 3a and 3b with the minor aforementioned modifications. We predicted that participants would estimate that the distance to a target was shorter when they intended to reach this target with a baton held in their right hand than without this baton. If tool-use effects rely on motor simulation, this difference should be smaller when performing a concurrent motor task (e.g., squeezing a ball) than when

\footnotetext{
${ }^{3}$ Random assignment consists of setting a known and equal probability to assign each participant to one group or another. Thus, some researchers have underlined that haphazard approaches like alternate assignment are not truly random even if it might seem to be at first sight (e.g., Beller et al., 2002, p. 566; Schulz \& Grimes, 2002, p. 515-516).

${ }^{4}$ This means that, all things being equal, in an infinite sequence of hypothetical replications of their studies, only $15 \%$ of replications will be able to detect the effect of interest. Moreover, according to the $95 \%$ CI of our average effect size the statistical power of their studies could be as low as .06 or as high as 33 .
} 
doing nothing. If motor simulation is effector-specific, performing the concurrent motor task should interfere with the tool availability effect only when performing it with the effector used to reach the target (i.e., right hand) and not with another effector (e.g., left foot). In contrast, if motor simulation is not effector-specific, performing the concurrent motor task should interfere with the tool availability effect regardless of the effector used to perform it.

\section{Method}

\section{Participants}

To determine a proper minimum sample size to detect the main effect of tool availability, we conducted an a priori power analysis using $\mathrm{G}^{*}$ Power 3 . Our power analysis indicated that a minimum of 93 participants was required to detect an effect size of Cohen's $\delta=0.34$ in a within-subject design with $90 \%$ power (two-tailed within-subject t-test, $\alpha=.05$ ). ${ }^{5}$

Accordingly, 93 participants from the University of Grenoble ( 82 women, $m_{\text {age }}=20.39, s_{\text {age }}=2.44$ ) took part in the study for course credit. They all reported having normal or corrected-to-normal vision and no neurological disorders. All participants provided written informed consent. The protocol followed the principles of the Declaration of Helsinki and the ethics principles of the American Psychological Association (2017). It was approved by the local ethics committee (CERNI, COMUE Univ. Grenoble Alpes, IRB00010290).

\section{Apparatus}

Participants sat approximately at $10 \mathrm{~cm}$ from the edge of a table (length $144 \mathrm{~cm}$, width $185 \mathrm{~cm}$, height $77 \mathrm{~cm}$ ). We developed the experimental program with E-prime 2.0. A downward facing projector displayed the stimuli on the table. A white circle $(\varnothing=3 \mathrm{~cm})$ placed at the edge of the table aligned with participants' mid-sagittal axis served as a reference point to estimate distances. Targets and comparison circles were also white circles $(\varnothing=1 \mathrm{~cm})$. Actual distances between the reference point and the target ranged from $74 \mathrm{~cm}$ to $119 \mathrm{~cm}$ $(74,79,84,89,94,99,104,109,114$, and $119 \mathrm{~cm})$. Depending on their experimental condition, participants used a 39-cm-long baton, which served as a reach-extending tool. We used two foam balls for the concurrent motor task, with one lying on the table and the other attached to a holder on the floor, depending on the nature of the interference.

\footnotetext{
${ }^{5}$ According to the $95 \%$ CI of our average effect size, a proper sample size for such a study could lie between $N=59$ and $N=184$.
}

\section{Procedure}

Each participant took part individually in this study. At the beginning of each trial, a cue indicated to the participants to start performing the motor concurrent task depending on their experimental condition (see below). Then, a target appeared and was immediately followed by two comparison circles projected at $5 \mathrm{~cm}$ on the left and right of the target. Participants had to estimate the distance between the reference point and the target with a visual-matching task. They adjusted the distance between the two comparison circles by pressing the keys 4 and 6 of a numeric keypad with their left hand until it matched the distance between the reference point and the target. After completing the estimation, both the target and the comparison circles disappeared, and the participant had to stop the motor-concurrent task and reach toward the previous location of the target.

Our experimental procedure is illustrated in Fig. 1. We manipulated the nature of the motor interference between subjects by randomly assigning the participants to one of two groups using E-Prime prior to beginning the experiment. For the hand-squeezing group, participants had to exert a constant squeeze on a rubber ball with their right hand while estimating the distance to the target. For the foot-squeezing group, participants had to exert a constant squeeze on the rubber ball with their left foot, as if it was a foot pedal, while estimating distance. In each motor interference group, we manipulated the tool availability within subjects through a block-wise variation. In the tool block, participants had to grab the baton with their right hand and reach to touch the target location immediately after the distance estimation. In the no-tool block, participants had to reach as far as they could and point to the target location immediately after the distance estimation as the target was always out of reach in this condition. Within each of these two blocks, we also manipulated the presence of motor interference within subjects through a block-wise variation. In the squeezing sub-block, participants had to exert a constant squeeze on the rubber ball with their right hand or their left foot, according to their group, while estimating the distance to the target. In the no-squeezing sub-block, participants had to keep their right hand on the table and their left foot on the floor while estimating the distance to the target. Contrary to Witt and Proffitt (2008), we chose to manipulate the tool availability and the presence of motor interference within subjects, as in previous studies (Bloesch et al., 2012; Costello et al., 2015; Witt et al., 2005), to obtain more statistical power.

Prior to each block, participants performed four practice trials to get used to the procedure. Once they felt comfortable with the instructions, participants completed all the test trials for the block, which made a total of 20 trials per sub-block (ten actual distances * two estimations). The trials were randomly presented and the block and sub-block orders were 


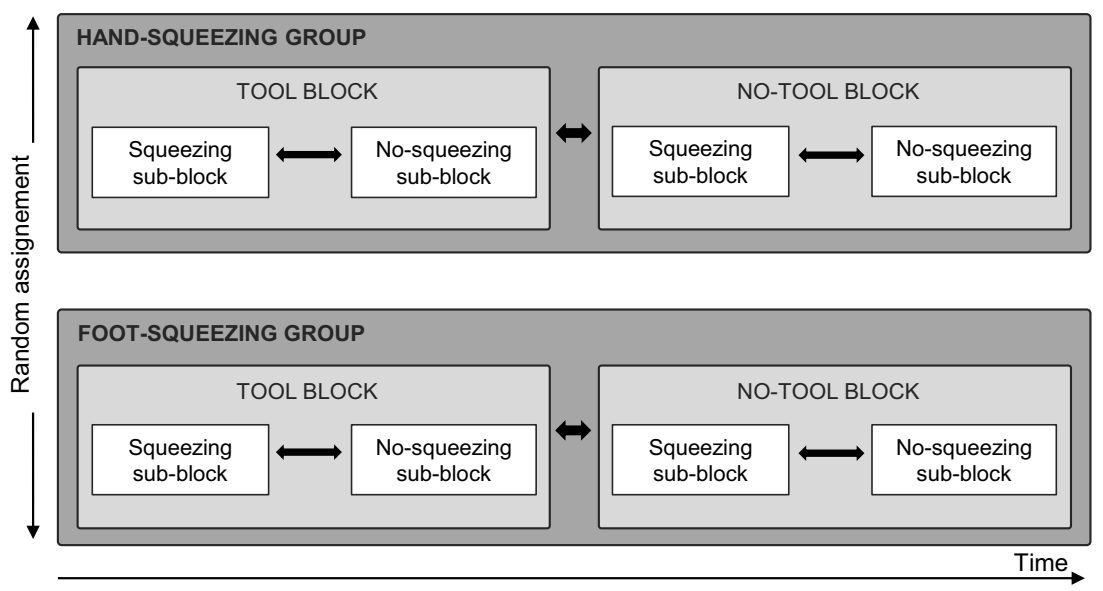

Fig. 1 Experimental procedure. Participants were randomly assigned to the hand- or foot-squeezing group. Each participant completed two counterbalanced blocks of trials: one with a tool, one without a tool.
Within each block, participants completed two counterbalanced subblocks: one with motor interference, one without motor interference counterbalanced. After completing the two blocks, the participants answered a questionnaire to assess their understanding of the hypothesis.

\section{Results}

We conducted our preliminary and main analyses according to a preregistered analysis plan. Full additional analyses are presented in the Supplementary Material. Raw data, R script, and Supplementary Material are available at https://osf.io/vp8sq/.

\section{Preliminary analyses}

For all our analyses, we checked the normality and homoscedasticity of the residuals by visually inspecting quantilequantile plots and plots of the residuals versus predicted values (Berry, 1993), respectively. We used visual inspection to detect the presence of outliers on the dependent variable (studentized deleted residuals) and their influence on our point estimates (Cook distance). To facilitate the visual inspection of the outliers, we considered data points with a studentized deleted residuals of 4 or greater as outliers. We detected no influential points with these methods. We removed trials for which participants validated their response before estimating the distance as indicated by estimations $\leq 10 \mathrm{~cm}(0.19 \%$ of the trials). We considered trials departing from individual mean by 2.5 standard deviations as outliers and we discarded them from the analysis $(0.23 \%$ of the trials).

\section{Main analyses}

Before collecting the data, we planned to conduct a contrast analysis focusing on the two contrasts that were of primary theoretical interest for our research question. ${ }^{6}$ We conducted this analysis with tool availability and the presence of motor interference as within-subject independent variables, the nature of the interference as between-subject independent variable, and distance estimation as dependent variable. Forty participants were in the foot-squeezing group and 53 participants were in the hand-squeezing group. The Presence of Motor Interference $\times$ Tool Availability interaction was not statistically significant, $t(91)=0.55, p=0.58$ (Table 1) and was not significantly moderated by the nature of the motor interference, $t(91)=0.87, p=0.39$, (Fig. 2).

\section{Additional analyses}

The difference between the tool and the no-tool conditions was not statistically significant, $m_{\text {no tool-tool }}=0.12 \mathrm{~cm}, s_{\text {no }}$ tool-tool $=6.4,95 \%$ CI $[-0.12,0.14], t(91)=1.50, p=0.14$. As we manipulated tool availability and the presence of motor interference within subjects in a block-wise manner (Fig. 1), the order of block presentation might have influenced participants' estimation. Among the participants who started with the no-squeezing block, participants first exposed to the tool block $\left(m_{\text {tool }}=104.21, s_{\text {tool }}=20.17\right)$ estimated that the target was $3.05 \mathrm{~cm}$ closer to them than participants first exposed to the no-tool block $\left(m_{\text {no tool }}=107.27, s_{\text {no tool }}=16.52\right), t(47)=$ $0.56, p=0.58$ (Fig. S1).

\footnotetext{
${ }^{6}$ As our independent variables had only two values, our test for the two interactions was exactly the same as the tests for the interaction in an omnibus ANOVA, which also tests effects that were not of primary interest to us. Readers interested in the full results of the ANOVA can look at the Table S2 in our Supplementary Material. This analysis also includes the actual distance as a within-subject independent variable. As we already had a complex research design, we did not include this independent variable in our main analyses because its variation was constant across the experimental conditions, we were not theoretically interested in its main effect on distance estimation, and we did not expect that it moderates our effects of interest.
} 
Table 1 Descriptive statistics for the tool availability and the presence of motor interference

\begin{tabular}{ccccrc}
\hline Tool availability & Presence of motor interference & $N$ & Mean distance estimation $(\mathrm{cm})$ & $S D$ & $95 \%$ CI \\
\hline NoTool & NoSqueezing & 93 & 105.79 & 19.43 & {$[101.79,109.78]$} \\
NoTool & Squeezing & 93 & 106.79 & 18.85 & {$[102.86,110.62]$} \\
Tool & NoSqueezing & 93 & 105.77 & 18.43 & {$[101.96,109.56]$} \\
Tool & Squeezing & 93 & 107.12 & 18.51 & {$[103.20,110.83]$} \\
\hline
\end{tabular}

Note. $N$ number of participants, $S D$ standard deviation, $C I$ confidence interval

We also checked whether (1) the presence of the motor interference affected at least the participants who showed the tool availability effect in the expected direction, (2) the effect of tool availability correlated with the participant's absolute estimation bias, and (3) discarding participants who guessed the tool was intended to affect them changed our conclusion. As indicated in our Supplementary Material, these analyses did not change our conclusions.

\section{Discussion}

This registered replication report (RRR) aimed to replicate the moderator effect of motor interference on tool availability effect on distance estimation suggested by Studies 3a and $3 b$ from Witt and Proffitt (2008). The tool availability effect is that extending one's arm reach with a tool makes out-of-reach targets appear closer (e.g., Osiurak et al., 2012; Witt \& Proffitt, 2008; Witt et al., 2005). According to Witt and Proffitt (2008), this effect should be "eliminated" when participants press a ball with their reaching hand during the distance estimation (p. 1486). Our results do not seem consistent with this predicted Presence of Interference $\times$
Tool Availability interaction (Fig. S5, top panel). However, a closer look at the data of Witt and Proffitt (2008) presented in our Supplementary Material also suggests that their results were also not that consistent with their prediction (Fig. S5, bottom right panel).

One could argue that detecting the Presence of the Motor Interference $\times$ Tool Availability interaction would have required a larger sample size than ours. While this is probably the case, this should not undermine the fact that we settled our sample size to have $90 \%$ power to detect at least the tool-use effect. Despite this, our tool-use effect was not statistically significant and was possibly too small, which might have produced a floor effect preventing us from observing the predicted interaction. This difficulty in replicating the tool-use effect is consistent with previous studies presented by their authors as failed replications of action-constraint effects on space perception (e.g., de Grave, Brenner, \& Smeets, 2011; Durgin \& Russell, 2008; Hutchison \& Loomis, 2006; Woods, Philbeck, \& Danoff, 2009).

One way to better estimate the tool availability effect would be to use within-subject designs more systematically in order to increase the precision of the estimation and, thus, statistical power. In addition, a within-subject design also allows estimation of

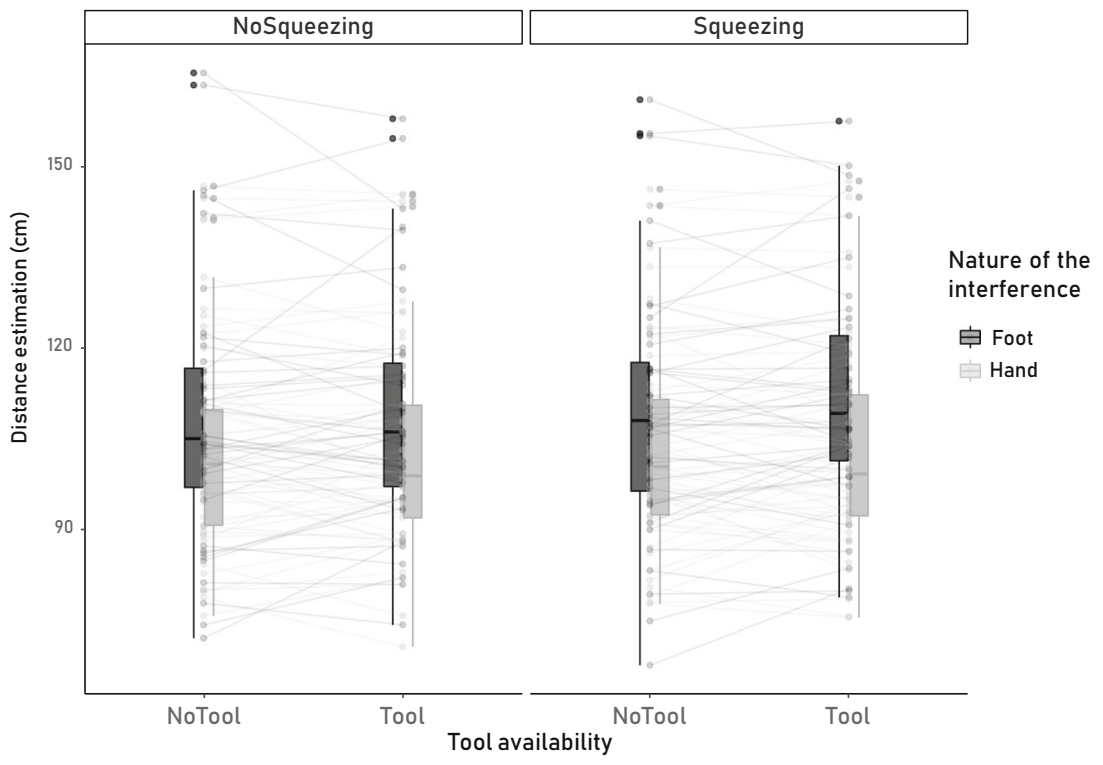

Fig. 2 Distance estimation as a function of the tool availability, the presence of the motor interference (panel), and the nature of the interference (gray shades). The central bar of the boxplot represents the median and the points show individual means. The lower and upper limits of the boxplot represent the first and third quartiles, respectively. The limits of the whiskers represent the 1.5 interquartile range 
an effect at the sample level as well as at the individual level (Miller \& Schwarz, 2018; see also Smith \& Little, 2018). This would be beneficial (1) to assess to what extent conclusions about a sample generalizes to the individuals who constitute it and (2) to identify individual differences to control for in priority to increase the effect size.

Another way to better estimate the tool-availability effect would be to develop a better measure of perceived distance. In the present study, we used the visual-matching task in which participants had to visually match a comparison distance to a target distance. Although Witt and Proffitt (2008) and others (e.g., Kirsch \& Kunde, 2013) have used this measure, it is prone to various response strategies that, if not controlled for, increase within- and between-participant variability. A good way to decrease this risk would be to measure perceived distance based on a task performed under time pressure.

This RRR aimed to advance the understanding of the action constraint effects on space perception by investigating more deeply one of their potential underlying mechanisms. The present study does not support Witt and Proffitt's (2008) hypothesis about the role of motor simulation. This is not to say that our RRR suggests the motor simulation account of action constraint effect on distance estimation can be dismissed entirely. Indeed, one replication, even with a relatively large sample size, calls for even more replications to ensure the reliability and cumulativeness of scientific results. The time has now come for researchers to combine their statistical power to move from single-lab to preregistered large-scale multi-lab replications. Only in such a way can we hope to convincingly elucidate the existence, the nature, the boundary conditions, and the underlying mechanisms of the action constraint effects on space perception.

\section{References}

American Psychological Association. (2017). Ethical principles of psychologists and code of conduct. Retrieved from http://www.apa.org/ethics/code/

Beller, E. M., Gebski, V., \& Keech, A. C. (2002). Randomisation in clinical trials. The Medical Journal of Australia, 177(10), 565-567.

Berry, W. D. (1993). Understanding regression assumptions. Newbury Park, CA: SAGE Publications.

Bloesch, E. K., Davoli, C. C., Roth, N., Brockmole, J. R., \& Abrams, R. a. (2012). Watch this! Observed tool use affects perceived distance. Psychonomic Bulletin \& Review, 19(2), 177-183. https://doi.org/10. 3758/s13423-011-0200-Z

Coello, Y., \& Delevoye-Turrell, Y. (2007). Embodiment, spatial categorisation and action. Consciousness and Cognition, 16(3), 667-683. https://doi.org/10.1016/j.concog.2007.07.003

Costello, M. C., Bloesch, E. K., Davoli, C. C., Panting, N. D., Abrams, R. A., \& Brockmole, J. R. (2015). Spatial representations in older adults are not modified by action: Evidence from tool use. Psychology and Aging, 30(3), 656-668. https://doi.org/10.1037/pag0000029

de Grave, D. D. J., Brenner, E., \& Smeets, J. B. J. (2011). Using a stick does not necessarily alter judged distances or reachability. PloS One, 6(2), e16697.

Durgin, F. H., \& Russell, R. C. (2008). Demand characteristics, not effort: The role of backpacks in judging distance and geographical slope.
Unpublished manuscript. Journal of Vision, 8(6). https://doi.org/10. $1167 / 8.6 .755$

Faul, F., Erdfelder, E., Lang, A.-G., \& Buchner, A. (2007). G*Power 3: A flexible statistical power program for the social, behavioral, and biomedical sciences. Behavior Research Methods, 39, 175-191.

Gelman, A., \& Stern, H. (2006). The Difference Between "Significant" and "not Significant" Is Not Itself Statistically Significant. The American Statistician, 60(4), 328-331. https://doi.org/10.1198/000313006X152649

Hutchison, J. J., \& Loomis, J. M. (2006). Does energy expenditure affect the perception of egocentric distance? A failure to replicate experiment 1 of Proffitt, Stefanucci, Banton, and Epstein (2003). The Spanish Journal of Psychology, 9(2), 332-339; discussion 340348. https://doi.org/10.1167/6.6.859

Jeannerod, M. (2001). Neural Simulation of Action: A Unifying Mechanism for Motor Cognition. Neuro, 14, 10-109. https://doi. org/10.1006/nimg.2001.0832

Kirsch, W., \& Kunde, W. (2013). Visual near space is scaled to parameters of current action plans. Journal of Experimental Psychology: Human Perception and Performance, 39(5), 1313-1325. https://doi. org/10.1037/a0031074

Miller, J., \& Schwarz, W. (2018). Implications of individual differences in on-average null effects. Journal of Experimental Psychology: General, 147(3), 377-397. https://doi.org/10.1037/xge0000367

Morgado, N., \& Palluel-Germain, R. (2016). How actions constrain the visual perception of space. In Y. Coello \& H. Fischer, Martin (Eds.), Foundations of embodied cognition (pp. 167-180). Routledge/ Taylor \& Francis Group.

Osiurak, F., Morgado, N., \& Palluel-Germain, R. (2012). Tool use and perceived distance: When unreachable becomes spontaneously reachable. Experimental Brain Research, 218(2), 331-339. https:// doi.org/10.1007/s00221-012-3036-5

Schulz, K. F., \& Grimes, D. A. (2002). Generation of allocation sequences in randomised trials: Chance, not choice. The Lancet, 359(9305), 515-519. https://doi.org/10.1016/S0140-6736(02)07683-3

Shadish, W. R., Cook, T. D., \& Campbell, T. (2002). Experimental and quasi-experimental designs for generalized causal inference. Boston, MA: Houghton Mifflin.

Smith, P. L., \& Little, D. R. (2018). Small is beautiful: In defense of the small-N design. Psychonomic Bulletin \& Review. https://doi.org/10. 3758/s13423-018-1451-8

Witt, J. K. (2011). Tool use influences perceived shape and perceived parallelism, which serve as indirect measures of perceived distance. Journal of Experimental Psychology. Human Perception and Performance, 37(4), 1148-1156. https://doi.org/10.1037/a0021933

Witt, J. K. (2017). A Role for Control in an Action-Specific Effect on Perception. Journal of Experimental Psychology: Human Perception and Performance.

Witt, J. K., \& Proffitt, D. R. (2008). Action-specific influences on distance perception: A role for motor simulation. Journal of Experimental Psychology. Human Perception and Performance, 34(6), 1479-1492. https://doi.org/10.1037/a0010781

Witt, J. K., Proffitt, D. R., \& Epstein, W. (2004). Perceiving distance: A role of effort and intent. Perception, 33(5), 577-590. https://doi.org/ $10.1068 / \mathrm{p} 5090$

Witt, J. K., Proffitt, D. R., \& Epstein, W. (2005). Tool use affects perceived distance, but only when you intend to use it. Journal of Experimental Psychology. Human Perception and Performance, 31(5), 880-888. https://doi.org/10.1037/0096-1523.31.5.880

Woods, A. J., Philbeck, J. W., \& Danoff, J. V. (2009). The various perceptions of distance: An alternative view of how effort affects distance judgments. Journal of Experimental Psychology: Human Perception and Performance, 35(4), 1104-1117. https://doi.org/10.1037/a0013622

Publisher's note Springer Nature remains neutral with regard to jurisdictional claims in published maps and institutional affiliations. 